CRYSTALLOGRAPHIC COMMUNICATIONS

ISSN 2056-9890

Received 7 October 2015

Accepted 17 November 2015

Edited by M. Gdaniec, Adam Mickiewicz University, Poland

Keywords: crystal structure; sulfonamide; hydrogen bond; chiral compound

CCDC reference: 1437453

Supporting information: this article has supporting information at journals.iucr.org/e

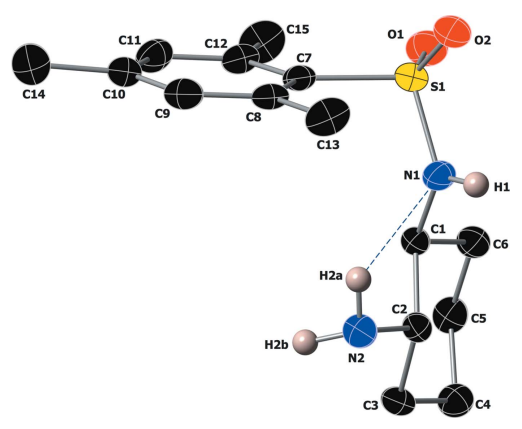

OPEN $\odot$ ACCESS

\section{Crystal structure of $N$-[(1S,2S)-2-aminocyclohexyl]- 2,4,6-trimethylbenzenesulfonamide}

\author{
Felix N. Ngassa, ${ }^{a *}$ Shannon M. Biros ${ }^{a}$ and Richard J. Staples ${ }^{b}$
}

aDepartment of Chemistry, Grand Valley State University, 1 Campus Dr., Allendale, MI 49401, USA, and ${ }^{\mathbf{b} C e n t e r}$ for Crystallographic Research, Department of Chemistry, Michigan State University, 578 S. Shaw Lane, East Lansing, MI 48824, USA. *Correspondence e-mail: ngassaf@gvsu.edu

The title compound, $\mathrm{C}_{15} \mathrm{H}_{24} \mathrm{~N}_{2} \mathrm{O}_{2} \mathrm{~S}$, was synthesized via a substitution reaction between the enantiopure $(1 S, 2 S)-(+)-1,2$-diaminocyclohexane and 2,4,6-trimethylbenzene-1-sulfonyl chloride. The cyclohexyl and phenyl substituents are oriented gauche around the sulfonamide $\mathrm{S}-\mathrm{N}$ bond. In the crystal, molecules are linked via $\mathrm{N}-\mathrm{H} \cdots \mathrm{N}$ hydrogen bonds, forming chains propagating along [100].

\section{Chemical context}

Many sulfonamides have been reported as anticancer, antiinflammatory, and antiviral agents (Navia, 2000; Yan et al., 2006; Palakurthy \& Mandal, 2011). The use of sulfonamides as catalysts in asymmetric synthesis has also been reported (Lao et al., 2009; Feng et al., 2010; Jin et al., 2010). Through explicit hydrogen-bonding interactions with specific functional groups, the electrophilicity and stereoselectivity of a given substrate is enhanced.

Conjugate addition reactions of aldehydes and ketones to nitroalkenes, catalyzed by chiral primary amines, have been reported (Huang \& Jacobsen, 2006; Rabalakos \& Wulff, 2008; Lao et al., 2009; Sun et al., 2012; Zhou et al., 2014; Ruiz-Olalla et al., 2015; Yang et al., 2015). The catalytic activity of chiral primary amine organocatalysts with particular emphasis on the role of the $\mathrm{N}-\mathrm{H}$ acidity and hydrogen bonding has also been investigated (Lao et al., 2009). Although the $\mathrm{N}-\mathrm{H}$ acidity and hydrogen-bonding modes could have an effect on the catalytic activity of the organocatalysts, the nature of the substrate and reaction conditions could be more important. Asymmetric conjugate addition reactions of aldehydes to nitroalkenes have also been reported as a convenient synthesis of $\gamma$-amino acids (Horne \& Gellman, 2008; Wiesner et al., 2008; Chi et al., 2008).<smiles>Cc1cc(C)c(S(=O)(=O)N[C@@H]2CCCC[C@H]2N)c(C)c1</smiles>

In line with our research interest in the synthesis of heterogeneous foldamers (Hayen et al., 2004), we synthesized the title compound as a chiral organocatalyst for conjugate addition. This conjugate addition was then applied for the 


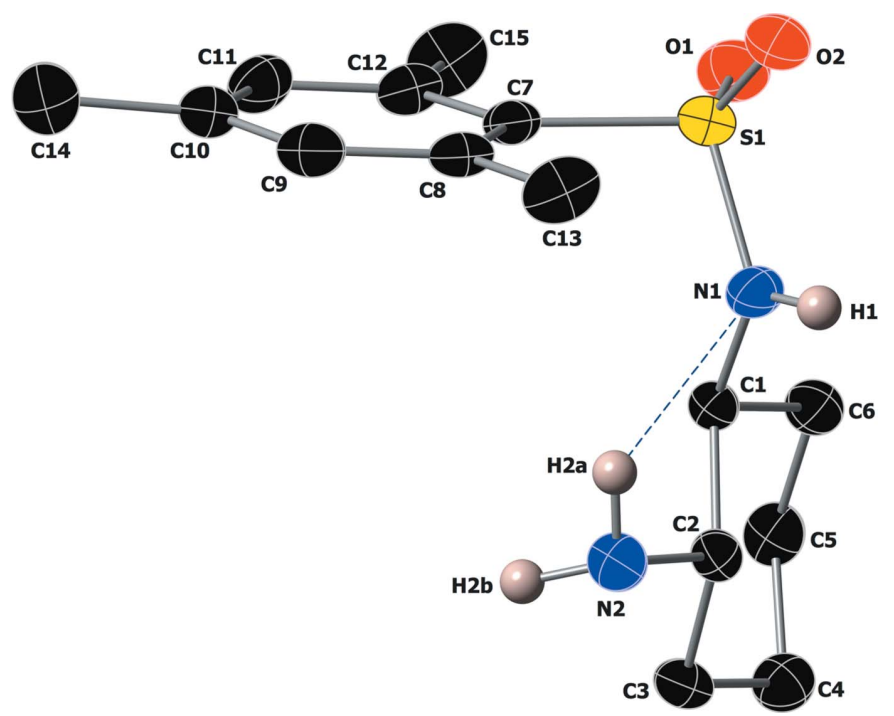

Figure 1

The asymmetric part of the unit cell along with the atom-numbering scheme and displacement ellipsoids drawn at the $50 \%$ probability level. An intramolecular $\mathrm{N}-\mathrm{H} \cdots \mathrm{N}$ interaction is shown with a blue dashed line. Only $\mathrm{N}-\mathrm{H}$ hydrogens are shown for clarity.

synthesis of $\gamma$-amino acids, which have been shown to be interesting foldamer building blocks (Horne \& Gellman, 2008). Therefore, as the title compound is of interest in our ongoing effort on foldamer design and synthesis, we report here on the synthesis and crystal structure of this chiral sulfonamide.

\section{Structural commentary}

The asymmetric part of the unit cell is shown in Fig. 1 along with the atom-numbering scheme. The absolute stereochemistry of this chiral sulfonamide was confirmed by a Flack parameter of 0.00 (2) (Parsons et al., 2013). The cyclohexyl (C1-C6) and benzene (C7-C12) substituents are oriented gauche around the sulfonamide $\mathrm{S}-\mathrm{N}$ bond, with a $\mathrm{C} 1-\mathrm{N} 1-$ $\mathrm{S} 1-\mathrm{C} 7$ torsion angle of $70.4(2)^{\circ}$. A weak intramolecular interaction is present between the amine $\mathrm{H} 2 \mathrm{~A}$ atom and the $s p^{2}$-hybridized sulfonamide N1 atom (Table 1).

As described in the Database survey section below, the structure of a racemic crystal of this compound has been reported (FAVHEP; Balsells, et al., 1998). In this crystal, there are two crystallographically unique molecules of the sulfonamide compound in the asymmetric unit. Here, the cyclohexyl and benzene substituents are oriented gauche around the $\mathrm{S}-$ $\mathrm{N}$ bond with torsion angles of 86.8 (8) and $69.1(7)^{\circ}$. While we expected that there would be an intramolecular hydrogen bond in this crystal, in the model deposited in the CSD there are no intramolecular hydrogen bonds present between the amine $\mathrm{N}-\mathrm{H}$ group and the sulfonamide $\mathrm{N}$ atom.

\section{Supramolecular features}

Molecules of the title compound are held together in the solid state by intermolecular hydrogen-bonding interactions
Table 1

Hydrogen-bond geometry $\left(\AA{ }^{\circ}\right)$.

\begin{tabular}{lllll}
\hline$D-\mathrm{H} \cdots A$ & $D-\mathrm{H}$ & $\mathrm{H} \cdots A$ & $D \cdots A$ & $D-\mathrm{H} \cdots A$ \\
\hline $\mathrm{N} 2-\mathrm{H} 2 A \cdots \mathrm{N} 1$ & $0.89(3)$ & $2.43(3)$ & $2.877(3)$ & $111(2)$ \\
$\mathrm{N} 1-\mathrm{H} 1 \cdots \mathrm{N} 2^{\mathrm{i}}$ & $0.79(3)$ & $2.14(3)$ & $2.921(3)$ & $170(3)$ \\
\hline
\end{tabular}

Symmetry code: (i) $x-\frac{1}{2},-y+\frac{3}{2},-z+1$.

between the donor sulfonamide $\mathrm{N} 1-\mathrm{H} 1$ and the acceptor amine N2 atoms (Table 1 and Fig. 2). These hydrogen bonds arrange molecules into supramolecular chains that are oriented along the [100] axis (Fig. 2). Weaker N2$\mathrm{H} 2 B \cdots \mathrm{O} 1(1+x, y, z)$ interactions with an $\mathrm{H} 2 B \cdots \mathrm{O} 1(1+x, y$, $z$ ) distance of $2.72 \AA$ between the donor amine $\mathrm{N} 2-\mathrm{H} 2 B$ and the acceptor sulfonamide $\mathrm{O} 1$ atoms can also be noticed within this chain.

As for the racemic crystal FAVHEP, in the model deposited in the CSD there is one intermolecular hydrogen bond present between a donor sulfonamide $\mathrm{N} 1-\mathrm{H} 1$ and a nearby amine

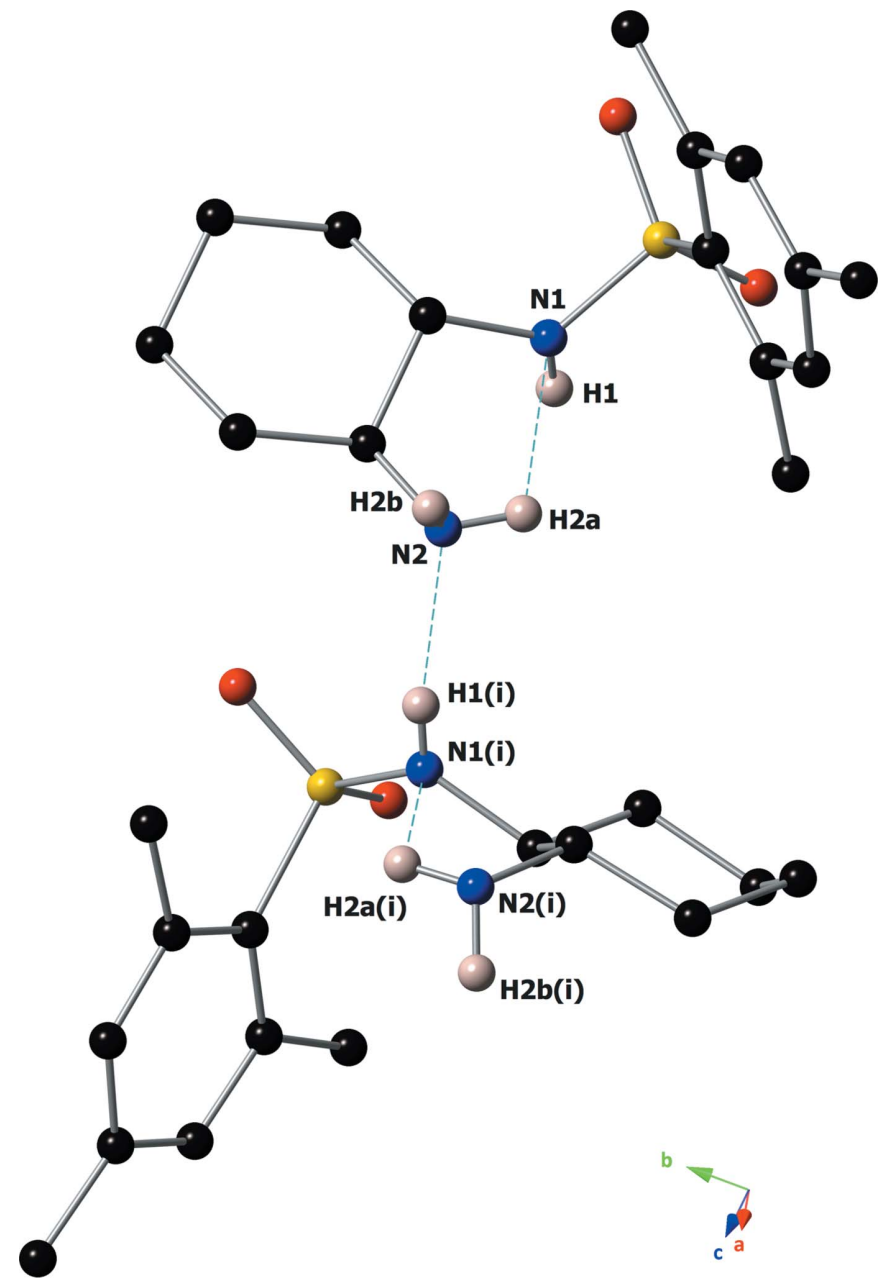

Figure 2

Intra- and intermolecular hydrogen-bonding interactions present in the crystal. Hydrogen bonds are drawn as blue dashed lines. Only $\mathrm{N}-\mathrm{H}$ hydrogens are shown for clarity. [Symmetry code: (i) $x-\frac{1}{2},-y+\frac{3}{2},-z+1$.] 
acceptor $\mathrm{N}$ atom $[D \cdots \mathrm{H}=0.860(7) \AA \AA \mathrm{H} \cdots A=2.160$ (8) $\mathrm{A}$; $D \cdots A=3.011(8) \AA ;$; $\left.D-\mathrm{H} \cdots A=169.9(5)^{\circ}\right]$.

\section{Database survey}

The Cambridge Structural Database (CSD, Version 5.36, May 2015; Groom \& Allen, 2014) contains 35 sulfonamides bearing a mesitylene group on the $\mathrm{S}$ atom. Of these, there are four structures where the substituent bonded to the sulfonamide $\mathrm{N}$ atom is an aliphatic six-membered ring. In structures RAWMAF (Hou et al., 2012) and ZIQPAS (Wu et al., 2014), the aminocyclohexane substituent is part of a larger fused-ring system. Interestingly, there are two structures with 1,2-diaminocyclohexane rings as the amide substituent. In structure OTOPAP (Schwarz et al., 2010), both amines of the trans-1,2diaminocyclohexane ring are bonded to a mesitylsulfonamide group. Structure FAVHEP (Balsells et al., 1998) is the same as the title compound, but is present as a racemic mixture that crystallized in the space group $P \overline{1}$.

\section{Synthesis and crystallization}

To a stirred solution of $(1 S, 2 S)-(+)-1,2$-diaminocyclohexane $(0.77 \mathrm{~g}, 6.74 \mathrm{mmol})$ in $5 \mathrm{ml}$ of $\mathrm{CH}_{2} \mathrm{Cl}_{2}$ at $273 \mathrm{~K}$ was added a solution of 2,4,6-trimethylbenzene-1-sulfonyl chloride ( $0.44 \mathrm{~g}$,

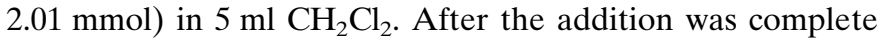
$(20 \mathrm{~min})$, the mixture was allowed to warm to room temperature and stirred overnight. The reaction mixture was washed with $\mathrm{H}_{2} \mathrm{O}(3 \times 25 \mathrm{ml})$ and the aqueous layer was backextracted with $\mathrm{CH}_{2} \mathrm{Cl}_{2}(20 \mathrm{ml})$. The combined organic extracts were dried over $\mathrm{Na}_{2} \mathrm{SO}_{4}$ and the solvent was removed under reduced pressure. The residue was purified by column chromatography over silica gel $\left(\mathrm{CH}_{2} \mathrm{Cl}_{2} /\right.$ EtOAc $\left.1: 1 \mathrm{v} / v\right)$ to afford a pale-yellow-white solid (yield: $0.46 \mathrm{~g}, 78 \%$ ). Part of the purified product was redissolved in $\mathrm{CH}_{2} \mathrm{Cl}_{2}$ and after slow evaporation for several days, white large chunky crystals (stained yellow) were formed that were suitable for analysis by X-ray diffraction (m.p. 406-407 K).

\section{Refinement}

Crystal data, data collection and structure refinement details are summarized in Table 2. The positions of all non-polar $\mathrm{H}$ atoms were calculated geometrically and refined to ride on their parent atoms, with $U_{\text {iso }}(\mathrm{H})=1.2 U_{\text {eq }}(\mathrm{C})$ for methine, methylene and aryl groups, and $U_{\text {iso }}(\mathrm{H})=1.5 U_{\text {eq }}(\mathrm{C})$ for methyl groups. $\mathrm{H}$ atoms bonded directly to $\mathrm{N}$ atoms (H1, $\mathrm{H} 2 \mathrm{~A}$ and $\mathrm{H} 2 B$ ) were located in difference-Fourier maps and refined isotropically.

\section{Acknowledgements}

The authors thank GVSU for financial support (Weldon Fund, CSCE), the NSF for a $300 \mathrm{MHz}$ Jeol FT-NMR (CCLI0087655) and Pfizer, Inc. for the donation of a Varian Inova 400 FT-NMR. The CCD-based X-ray diffractometers at
Table 2

Experimental details.

\begin{tabular}{|c|c|}
\hline \multicolumn{2}{|l|}{ Crystal data } \\
\hline Chemical formula & $\mathrm{C}_{15} \mathrm{H}_{24} \mathrm{~N}_{2} \mathrm{O}_{2} \mathrm{~S}$ \\
\hline$M_{\mathrm{r}}$ & 296.42 \\
\hline Crystal system, space group & Orthorhombic, $P 2_{1} 2_{1} 2_{1}$ \\
\hline Temperature $(\mathrm{K})$ & 173 \\
\hline$a, b, c(\AA)$ & $\begin{array}{l}6.5215(4), 10.0202(6), \\
23.3660(15)\end{array}$ \\
\hline$V\left(\AA^{3}\right)$ & $1526.89(16)$ \\
\hline$Z$ & 4 \\
\hline Radiation type & Mo $K \alpha$ \\
\hline$\mu\left(\mathrm{mm}^{-1}\right)$ & 0.22 \\
\hline Crystal size $(\mathrm{mm})$ & $0.37 \times 0.20 \times 0.15$ \\
\hline \multicolumn{2}{|l|}{ Data collection } \\
\hline Diffractometer & Bruker APEXII CCD \\
\hline Absorption correction & $\begin{array}{l}\text { Multi-scan (SADABS; Bruker, } \\
\text { 2014) }\end{array}$ \\
\hline$T_{\min }, T_{\max }$ & $0.706,0.745$ \\
\hline $\begin{array}{l}\text { No. of measured, independent and } \\
\text { observed }[I>2 \sigma(I)] \text { reflections }\end{array}$ & 25587, 2799, 2667 \\
\hline$R_{\text {int }}$ & 0.034 \\
\hline$(\sin \theta / \lambda)_{\max }\left(\AA^{-1}\right)$ & 0.602 \\
\hline \multicolumn{2}{|l|}{ Refinement } \\
\hline$R\left[F^{2}>2 \sigma\left(F^{2}\right)\right], w R\left(F^{2}\right), S$ & $0.029,0.071,1.06$ \\
\hline No. of reflections & 2799 \\
\hline No. of parameters & 196 \\
\hline $\mathrm{H}$-atom treatment & $\begin{array}{l}\mathrm{H} \text { atoms treated by a mixture of } \\
\text { independent and constrained } \\
\text { refinement }\end{array}$ \\
\hline$\Delta \rho_{\max }, \Delta \rho_{\min }\left(\mathrm{e} \AA^{-3}\right)$ & $0.19,-0.21$ \\
\hline Absolute structure & $\begin{array}{l}\text { Flack parameter } x \text { determined } \\
\text { using } 1098 \text { quotients } \\
{\left[\left(I^{+}\right)-\left(I^{-}\right)\right] /\left[\left(I^{+}\right)+\left(I^{-}\right)\right]} \\
(\text {Parsons } \text { et al. }, \text { 2013) }\end{array}$ \\
\hline Absolute structure parameter & $0.00(2)$ \\
\hline
\end{tabular}

Computer programs: APEX2 ad SAINT (Bruker, 2013), SHELXS97 (Sheldrick, 2008), SHELXL2014 (Sheldrick, 2015), OLEX2 (Dolomanov et al., 2009; Bourhis et al., 2015) and CrystalMaker (Palmer, 2007).

Michigan State University were upgraded and/or replaced by departmental funds.

\section{References}

Balsells, J., Mejorado, L., Phillips, M., Ortega, F., Aguirre, G., Somanathan, R. \& Walsh, P. J. (1998). Tetrahedron Asymmetry, 9, 4135-4142.

Bourhis, L. J., Dolomanov, O. V., Gildea, R. J., Howard, J. A. K. \& Puschmann, H. (2015). Acta Cryst. A71, 59-75.

Bruker (2013). APEX2 and SAINT. Bruker AXS Inc., Madison, Wisconsin, USA.

Bruker (2014). SADABS. Bruker AXS Inc., Madison, Wisconsin, USA.

Chi, Y., Guo, L., Kopf, N. A. \& Gellman, S. H. (2008). J. Am. Chem. Soc. 130, 5608-5609.

Dolomanov, O. V., Bourhis, L. J., Gildea, R. J., Howard, J. A. K. \& Puschmann, H. (2009). J. Appl. Cryst. 42, 339-341.

Feng, Y., Xiaomin, S., Zhichao, J., Shiganag, W., Xinmiao, L. \& Jinxing, Y. (2010). Chem Commun. pp. 4589-4590.

Groom, C. R. \& Allen, F. H. (2014). Angew. Chem. Int. Ed. 53, 662671.

Hayen, A., Schmitt, M. N., Ngassa, F. N., Thomasson, K. A. \& Gellman, S. H. (2004). Angew. Chem. Int. Ed. 43, 505-510.

Horne, W. S. \& Gellman, S. H. (2008). Acc. Chem. Res. 41, 1399-1408.

Hou, W., Zheng, B., Chen, J. \& Peng, Y. (2012). Org. Lett. 14, 23782381. 
Huang, H. \& Jacobsen, E. N. (2006). J. Am. Chem. Soc. 128, 71707171.

Jin, W., Li, X., Huang, Y., Wu, F. \& Wan, B. (2010). Chem. Eur. J. 16, 8259-8261.

Lao, J., Zhang, X., Wang, J., Li, X., Yan, M. \& Luo, H. (2009). Tetrahedron Asymmetry, 20, 2818-2822.

Navia, M. A. (2000). Science, 288, 2132-2133.

Palakurthy, N. B. \& Mandal, B. (2011). Tetrahedron Lett. 52, 71327134.

Palmer, D. (2007). CrystalMaker. Crystal Maker Software, Bicester, England.

Parsons, S., Flack, H. D. \& Wagner, T. (2013). Acta Cryst. B69, 249259.

Rabalakos, C. \& Wulff, W. D. (2008). J. Am. Chem. Soc. 130, 1352413525.

Ruiz-Olalla, A., Retamosa, M. de G. \& Cossío, F. P. (2015). J. Org. Chem. 80, 5588-5599.
Schwarz, A. D., Herbert, K. R., Paniagua, C. \& Mountford, P. (2010). Organometallics, 29, 4171-4188.

Sheldrick, G. M. (2008). Acta Cryst. A64, 112-122.

Sheldrick, G. M. (2015). Acta Cryst. C71, 3-8.

Sun, Z. W., Peng, F. Z., Li, Z. Q., Zou, L. W., Zhang, S. X., Li, X. \& Shao, Z. H. (2012). J. Org. Chem. 77, 4103-4110.

Wiesner, M., Revell, J. D., Tonazzi, S. \& Wennemers, H. (2008). J. Am. Chem. Soc. 130, 5610-5611.

Wu, L., Wang, Y., Song, H., Tang, L., Zhou, Z. \& Tang, C. (2014). ChemCatChem, 6, 649-654.

Yan, L., Bertarelli, D. C., Hayallah, A. M., Meyer, H., Klotz, K. N. \& Müller, C. E. (2006). J. Med. Chem. 49, 4384-4391.

Yang, D., Li, D., Wang, L., Zhao, D. \& Wang, R. (2015). J. Org. Chem. 80, 4336-4348.

Zhou, Z., Feng, X., Yin, X. \& Chen, Y. C. (2014). Org. Lett. 16, 2370 2373. 


\section{supporting information}

Acta Cryst. (2015). E71, 1521-1524 [https://doi.org/10.1107/S205698901502191X]

\section{Crystal structure of $\mathrm{N}$-[(1S,2S)-2-aminocyclohexyl]-2,4,6-trimethylbenzene- sulfonamide}

\section{Felix N. Ngassa, Shannon M. Biros and Richard J. Staples}

Computing details

Data collection: APEX2 (Bruker, 2013); cell refinement: SAINT (Bruker, 2013); data reduction: SAINT (Bruker, 2013); program(s) used to solve structure: SHELXS97 (Sheldrick, 2008); program(s) used to refine structure: SHELXL2014 (Sheldrick, 2015); molecular graphics: OLEX2 (Dolomanov et al., 2009; Bourhis et al., 2015); software used to prepare material for publication: CrystalMaker (Palmer, 2007).

$N$-[(1S,2S)-2-Aminocyclohexyl]-2,4,6-trimethylbenzenesulfonamide

\section{Crystal data}

$\mathrm{C}_{15} \mathrm{H}_{24} \mathrm{~N}_{2} \mathrm{O}_{2} \mathrm{~S}$

$M_{r}=296.42$

Orthorhombic, $P 2_{1} 2_{1} 2_{1}$

$a=6.5215(4) \AA$

$b=10.0202(6) \AA$

$c=23.3660(15) \AA$

$V=1526.89(16) \AA^{3}$

$Z=4$

$F(000)=640$

\section{Data collection}

Bruker APEXII CCD diffractometer

Radiation source: sealed tube Graphite monochromator Detector resolution: 8 pixels $\mathrm{mm}^{-1}$ $\varphi$ and $\omega$ scans

Absorption correction: multi-scan

(SADABS; Bruker, 2014)

$T_{\min }=0.706, T_{\max }=0.745$

Refinement

Refinement on $F^{2}$

Least-squares matrix: full

$R\left[F^{2}>2 \sigma\left(F^{2}\right)\right]=0.029$

$w R\left(F^{2}\right)=0.071$

$S=1.06$

2799 reflections

196 parameters

0 restraints

Hydrogen site location: mixed
$D_{\mathrm{x}}=1.289 \mathrm{Mg} \mathrm{m}^{-3}$

Mo $K \alpha$ radiation, $\lambda=0.71073 \AA$

Cell parameters from 9968 reflections

$\theta=2.2-25.3^{\circ}$

$\mu=0.22 \mathrm{~mm}^{-1}$

$T=173 \mathrm{~K}$

Block, colourless

$0.37 \times 0.20 \times 0.15 \mathrm{~mm}$

25587 measured reflections

2799 independent reflections

2667 reflections with $I>2 \sigma(I)$

$R_{\text {int }}=0.034$

$\theta_{\max }=25.4^{\circ}, \theta_{\min }=1.7^{\circ}$

$h=-7 \rightarrow 7$

$k=-12 \rightarrow 12$

$l=-28 \rightarrow 28$

$\mathrm{H}$ atoms treated by a mixture of independent and constrained refinement

$w=1 /\left[\sigma^{2}\left(F_{\mathrm{o}}^{2}\right)+(0.0313 P)^{2}+0.5049 P\right]$

where $P=\left(F_{\mathrm{o}}^{2}+2 F_{\mathrm{c}}{ }^{2}\right) / 3$

$(\Delta / \sigma)_{\max }<0.001$

$\Delta \rho_{\max }=0.19 \mathrm{e} \AA^{-3}$

$\Delta \rho_{\min }=-0.21$ e $\AA^{-3}$ 
Absolute structure: Flack parameter $x$ determined using 1098 quotients $\left[\left(I^{+}\right)-\left(I^{-}\right)\right] /\left[\left(I^{+}\right)+(I)\right]($ Parsons et al., 2013)

Absolute structure parameter: $0.00(2)$

Special details

Geometry. All e.s.d.'s (except the e.s.d. in the dihedral angle between two 1.s. planes) are estimated using the full covariance matrix. The cell e.s.d.'s are taken into account individually in the estimation of e.s.d.'s in distances, angles and torsion angles; correlations between e.s.d.'s in cell parameters are only used when they are defined by crystal symmetry. An approximate (isotropic) treatment of cell e.s.d.'s is used for estimating e.s.d.'s involving l.s. planes.

Fractional atomic coordinates and isotropic or equivalent isotropic displacement parameters $\left(\AA^{2}\right)$

\begin{tabular}{|c|c|c|c|c|}
\hline & $x$ & $y$ & $z$ & $U_{\text {iso }} * / U_{\text {eq }}$ \\
\hline S1 & $0.39425(9)$ & $0.81200(6)$ & $0.62892(2)$ & $0.03018(16)$ \\
\hline $\mathrm{O} 1$ & $0.2464(3)$ & $0.72704(19)$ & $0.65596(8)$ & $0.0412(4)$ \\
\hline $\mathrm{O} 2$ & $0.3238(3)$ & 0.93797 (17) & $0.60697(8)$ & $0.0408(5)$ \\
\hline N1 & $0.5015(3)$ & $0.7361(2)$ & $0.57616(9)$ & $0.0297(5)$ \\
\hline $\mathrm{N} 2$ & $0.9140(3)$ & $0.6769(2)$ & $0.54061(8)$ & $0.0287(4)$ \\
\hline $\mathrm{C} 1$ & $0.5776(3)$ & $0.5984(2)$ & $0.58084(9)$ & $0.0238(5)$ \\
\hline $\mathrm{H} 1 \mathrm{~A}$ & 0.6425 & 0.5872 & 0.6193 & $0.029 *$ \\
\hline $\mathrm{C} 2$ & $0.7442(3)$ & $0.5816(2)$ & $0.53502(9)$ & $0.0234(5)$ \\
\hline $\mathrm{H} 2$ & 0.6780 & 0.5986 & 0.4971 & $0.028^{*}$ \\
\hline $\mathrm{C} 3$ & $0.8271(3)$ & $0.4398(2)$ & $0.53360(10)$ & $0.0273(5)$ \\
\hline $\mathrm{H} 3 \mathrm{~A}$ & 0.9052 & 0.4225 & 0.5692 & $0.033^{*}$ \\
\hline $\mathrm{H} 3 \mathrm{~B}$ & 0.9228 & 0.4306 & 0.5009 & $0.033^{*}$ \\
\hline $\mathrm{C} 4$ & $0.6574(4)$ & $0.3363(2)$ & $0.52793(10)$ & $0.0308(5)$ \\
\hline $\mathrm{H} 4 \mathrm{~A}$ & 0.7174 & 0.2457 & 0.5300 & $0.037 *$ \\
\hline $\mathrm{H} 4 \mathrm{~B}$ & 0.5902 & 0.3460 & 0.4902 & $0.037 *$ \\
\hline $\mathrm{C} 5$ & $0.4980(4)$ & $0.3528(2)$ & $0.57518(11)$ & $0.0314(5)$ \\
\hline H5A & 0.3864 & 0.2871 & 0.5696 & $0.038^{*}$ \\
\hline $\mathrm{H} 5 \mathrm{~B}$ & 0.5624 & 0.3353 & 0.6128 & $0.038^{*}$ \\
\hline C6 & 0.4094 (4) & $0.4934(2)$ & $0.57442(10)$ & $0.0300(5)$ \\
\hline H6A & 0.3095 & 0.5031 & 0.6061 & $0.036^{*}$ \\
\hline H6B & 0.3356 & 0.5080 & 0.5379 & $0.036^{*}$ \\
\hline $\mathrm{C} 7$ & $0.5988(4)$ & $0.8413(2)$ & $0.67770(9)$ & $0.0252(5)$ \\
\hline $\mathrm{C} 8$ & $0.7523(4)$ & $0.9336(2)$ & $0.66163(9)$ & $0.0270(5)$ \\
\hline C9 & $0.9071(4)$ & $0.9619(2)$ & $0.69984(10)$ & $0.0321(5)$ \\
\hline H9 & 1.0119 & 1.0225 & 0.6887 & $0.039 *$ \\
\hline $\mathrm{C} 10$ & 0.9154 (4) & $0.9054(2)$ & $0.75383(10)$ & $0.0340(6)$ \\
\hline $\mathrm{C} 11$ & $0.7662(4)$ & $0.8131(3)$ & $0.76822(10)$ & $0.0346(5)$ \\
\hline H11 & 0.7724 & 0.7723 & 0.8049 & $0.042 *$ \\
\hline C12 & $0.6078(4)$ & 0.7775 (2) & $0.73148(9)$ & $0.0291(5)$ \\
\hline C13 & $0.7568(5)$ & $1.0087(2)$ & $0.60541(10)$ & $0.0384(6)$ \\
\hline H13A & 0.7084 & 0.9502 & 0.5746 & $0.058^{*}$ \\
\hline H13B & 0.8974 & 1.0373 & 0.5972 & $0.058^{*}$ \\
\hline $\mathrm{H} 13 \mathrm{C}$ & 0.6675 & 1.0871 & 0.6080 & $0.058^{*}$ \\
\hline C14 & $1.0799(5)$ & $0.9459(3)$ & $0.79566(13)$ & $0.0532(8)$ \\
\hline H14A & 1.2117 & 0.9523 & 0.7757 & $0.080 *$ \\
\hline
\end{tabular}


supporting information

$\begin{array}{lllll}\text { H14B } & 1.0897 & 0.8789 & 0.8261 & 0.080^{*} \\ \text { H14C } & 1.0452 & 1.0327 & 0.8124 & 0.080^{*} \\ \text { C15 } & 0.4607(5) & 0.6726(3) & 0.75300(12) & 0.0440(7) \\ \text { H15A } & 0.3236 & 0.7116 & 0.7569 & 0.066^{*} \\ \text { H15B } & 0.5071 & 0.6399 & 0.7903 & 0.066^{*} \\ \text { H15C } & 0.4559 & 0.5983 & 0.7258 & 0.066^{*} \\ \text { H2A } & 0.864(5) & 0.754(3) & 0.5537(12) & 0.047(8)^{*} \\ \text { H1 } & 0.484(5) & 0.768(3) & 0.5458(13) & 0.040(8)^{*} \\ \text { H2B } & 1.003(5) & 0.645(3) & 0.5683(13) & 0.045(8)^{*}\end{array}$

Atomic displacement parameters $\left(\AA^{2}\right)$

\begin{tabular}{lllllll}
\hline & $U^{11}$ & $U^{22}$ & $U^{33}$ & $U^{12}$ & $U^{13}$ & $U^{23}$ \\
\hline S1 & $0.0271(3)$ & $0.0327(3)$ & $0.0307(3)$ & $0.0072(3)$ & $0.0005(3)$ & $-0.0065(2)$ \\
O1 & $0.0275(8)$ & $0.0496(11)$ & $0.0465(10)$ & $-0.0037(8)$ & $0.0092(8)$ & $-0.0111(8)$ \\
O2 & $0.0427(10)$ & $0.0387(10)$ & $0.0410(10)$ & $0.0196(8)$ & $-0.0057(8)$ & $-0.0067(8)$ \\
N1 & $0.0371(11)$ & $0.0293(11)$ & $0.0228(10)$ & $0.0109(9)$ & $-0.0014(9)$ & $-0.0010(9)$ \\
N2 & $0.0283(10)$ & $0.0250(10)$ & $0.0329(10)$ & $-0.0021(9)$ & $0.0002(9)$ & $-0.0004(9)$ \\
C1 & $0.0239(11)$ & $0.0250(11)$ & $0.0225(10)$ & $0.0044(9)$ & $-0.0012(9)$ & $-0.0009(9)$ \\
C2 & $0.0228(10)$ & $0.0238(11)$ & $0.0236(10)$ & $0.0009(9)$ & $0.0001(9)$ & $0.0016(9)$ \\
C3 & $0.0261(11)$ & $0.0259(12)$ & $0.0300(12)$ & $0.0053(10)$ & $0.0036(9)$ & $0.0003(9)$ \\
C4 & $0.0357(13)$ & $0.0241(11)$ & $0.0326(12)$ & $0.0008(10)$ & $0.0001(10)$ & $-0.0010(9)$ \\
C5 & $0.0315(13)$ & $0.0313(13)$ & $0.0315(12)$ & $-0.0059(10)$ & $0.0014(10)$ & $0.0010(10)$ \\
C6 & $0.0229(11)$ & $0.0359(12)$ & $0.0312(11)$ & $-0.0006(11)$ & $0.0032(11)$ & $-0.0033(10)$ \\
C7 & $0.0278(11)$ & $0.0242(10)$ & $0.0235(10)$ & $0.0038(10)$ & $0.0040(10)$ & $-0.0040(8)$ \\
C8 & $0.0327(12)$ & $0.0217(11)$ & $0.0267(11)$ & $0.0030(10)$ & $0.0081(10)$ & $-0.0020(9)$ \\
C9 & $0.0291(12)$ & $0.0290(12)$ & $0.0382(13)$ & $-0.0015(11)$ & $0.0069(12)$ & $-0.0045(10)$ \\
C10 & $0.0319(13)$ & $0.0361(13)$ & $0.0340(13)$ & $0.0076(11)$ & $-0.0008(11)$ & $-0.0102(10)$ \\
C11 & $0.0465(14)$ & $0.0342(12)$ & $0.0232(11)$ & $0.0075(13)$ & $0.0014(11)$ & $0.0010(10)$ \\
C12 & $0.0367(12)$ & $0.0247(11)$ & $0.0259(11)$ & $0.0018(11)$ & $0.0062(11)$ & $-0.0010(9)$ \\
C13 & $0.0536(16)$ & $0.0285(12)$ & $0.0331(13)$ & $-0.0021(12)$ & $0.0098(13)$ & $0.0058(11)$ \\
C14 & $0.0455(18)$ & $0.0629(19)$ & $0.0512(17)$ & $0.0037(16)$ & $-0.0124(16)$ & $-0.0140(15)$ \\
C15 & $0.0569(18)$ & $0.0351(14)$ & $0.0401(14)$ & $-0.0079(13)$ & $0.0094(13)$ & $0.0066(12)$ \\
& & & & & &
\end{tabular}

Geometric parameters $\left(\AA,^{\circ}\right)$

\begin{tabular}{llll}
\hline $\mathrm{S} 1-\mathrm{O} 1$ & $1.4330(19)$ & $\mathrm{C} 6-\mathrm{H} 6 \mathrm{~A}$ & 0.9900 \\
$\mathrm{~S} 1-\mathrm{O} 2$ & $1.4379(18)$ & $\mathrm{C} 6-\mathrm{H} 6 \mathrm{~B}$ & 0.9900 \\
$\mathrm{~S} 1-\mathrm{N} 1$ & $1.609(2)$ & $\mathrm{C} 7-\mathrm{C} 8$ & $1.414(3)$ \\
$\mathrm{S} 1-\mathrm{C} 7$ & $1.779(2)$ & $\mathrm{C} 7-\mathrm{C} 12$ & $1.411(3)$ \\
$\mathrm{N} 1-\mathrm{C} 1$ & $1.470(3)$ & $\mathrm{C} 8-\mathrm{C} 9$ & $1.377(3)$ \\
$\mathrm{N} 1-\mathrm{H} 1$ & $0.79(3)$ & $\mathrm{C} 8-\mathrm{C} 13$ & $1.514(3)$ \\
$\mathrm{N} 2-\mathrm{C} 2$ & $1.469(3)$ & $\mathrm{C} 9-\mathrm{H} 9$ & 0.9500 \\
$\mathrm{~N} 2-\mathrm{H} 2 \mathrm{~A}$ & $0.89(3)$ & $\mathrm{C} 9-\mathrm{C} 10$ & $1.384(3)$ \\
$\mathrm{N} 2-\mathrm{H} 2 \mathrm{~B}$ & $0.93(3)$ & $\mathrm{C} 10-\mathrm{C} 11$ & $1.384(4)$ \\
$\mathrm{C} 1-\mathrm{H} 1 \mathrm{~A}$ & 1.0000 & $\mathrm{C} 10-\mathrm{C} 14$ & $1.507(4)$ \\
$\mathrm{C} 1-\mathrm{C} 2$ & $1.535(3)$ & $\mathrm{C} 11-\mathrm{H} 11$ & 0.9500 \\
$\mathrm{C} 1-\mathrm{C} 6$ & $1.527(3)$ & $\mathrm{C} 11-\mathrm{C} 12$ & $1.390(4)$
\end{tabular}




\begin{tabular}{|c|c|c|c|}
\hline $\mathrm{C} 2-\mathrm{H} 2$ & 1.0000 & $\mathrm{C} 12-\mathrm{C} 15$ & $1.509(3)$ \\
\hline $\mathrm{C} 2-\mathrm{C} 3$ & $1.521(3)$ & $\mathrm{C} 13-\mathrm{H} 13 \mathrm{~A}$ & 0.9800 \\
\hline $\mathrm{C} 3-\mathrm{H} 3 \mathrm{~A}$ & 0.9900 & C13-H13B & 0.9800 \\
\hline C $3-\mathrm{H} 3 \mathrm{~B}$ & 0.9900 & $\mathrm{C} 13-\mathrm{H} 13 \mathrm{C}$ & 0.9800 \\
\hline $\mathrm{C} 3-\mathrm{C} 4$ & $1.522(3)$ & $\mathrm{C} 14-\mathrm{H} 14 \mathrm{~A}$ & 0.9800 \\
\hline $\mathrm{C} 4-\mathrm{H} 4 \mathrm{~A}$ & 0.9900 & C14-H14B & 0.9800 \\
\hline $\mathrm{C} 4-\mathrm{H} 4 \mathrm{~B}$ & 0.9900 & $\mathrm{C} 14-\mathrm{H} 14 \mathrm{C}$ & 0.9800 \\
\hline $\mathrm{C} 4-\mathrm{C} 5$ & $1.525(3)$ & C15-H15A & 0.9800 \\
\hline $\mathrm{C} 5-\mathrm{H} 5 \mathrm{~A}$ & 0.9900 & C15-H15B & 0.9800 \\
\hline C5-H5B & 0.9900 & $\mathrm{C} 15-\mathrm{H} 15 \mathrm{C}$ & 0.9800 \\
\hline $\mathrm{C} 5-\mathrm{C} 6$ & $1.523(3)$ & & \\
\hline $\mathrm{O} 1-\mathrm{S} 1-\mathrm{O} 2$ & $117.62(12)$ & $\mathrm{C} 1-\mathrm{C} 6-\mathrm{H} 6 \mathrm{~A}$ & 109.4 \\
\hline $\mathrm{O} 1-\mathrm{S} 1-\mathrm{N} 1$ & $110.46(11)$ & $\mathrm{C} 1-\mathrm{C} 6-\mathrm{H} 6 \mathrm{~B}$ & 109.4 \\
\hline $\mathrm{O} 1-\mathrm{S} 1-\mathrm{C} 7$ & $108.68(11)$ & $\mathrm{C} 5-\mathrm{C} 6-\mathrm{C} 1$ & 111.33 (19) \\
\hline $\mathrm{O} 2-\mathrm{S} 1-\mathrm{N} 1$ & $106.31(11)$ & $\mathrm{C} 5-\mathrm{C} 6-\mathrm{H} 6 \mathrm{~A}$ & 109.4 \\
\hline $\mathrm{O} 2-\mathrm{S} 1-\mathrm{C} 7$ & $108.86(11)$ & $\mathrm{C} 5-\mathrm{C} 6-\mathrm{H} 6 \mathrm{~B}$ & 109.4 \\
\hline $\mathrm{N} 1-\mathrm{S} 1-\mathrm{C} 7$ & $104.06(11)$ & $\mathrm{H} 6 \mathrm{~A}-\mathrm{C} 6-\mathrm{H} 6 \mathrm{~B}$ & 108.0 \\
\hline $\mathrm{S} 1-\mathrm{N} 1-\mathrm{H} 1$ & $116(2)$ & $\mathrm{C} 8-\mathrm{C} 7-\mathrm{S} 1$ & $117.96(16)$ \\
\hline $\mathrm{C} 1-\mathrm{N} 1-\mathrm{S} 1$ & $122.24(17)$ & $\mathrm{C} 12-\mathrm{C} 7-\mathrm{S} 1$ & $121.78(19)$ \\
\hline $\mathrm{C} 1-\mathrm{N} 1-\mathrm{H} 1$ & $120(2)$ & $\mathrm{C} 12-\mathrm{C} 7-\mathrm{C} 8$ & $120.2(2)$ \\
\hline $\mathrm{C} 2-\mathrm{N} 2-\mathrm{H} 2 \mathrm{~A}$ & $108(2)$ & $\mathrm{C} 7-\mathrm{C} 8-\mathrm{C} 13$ & $124.7(2)$ \\
\hline $\mathrm{C} 2-\mathrm{N} 2-\mathrm{H} 2 \mathrm{~B}$ & $108.1(18)$ & $\mathrm{C} 9-\mathrm{C} 8-\mathrm{C} 7$ & $118.8(2)$ \\
\hline $\mathrm{H} 2 \mathrm{~A}-\mathrm{N} 2-\mathrm{H} 2 \mathrm{~B}$ & $107(3)$ & $\mathrm{C} 9-\mathrm{C} 8-\mathrm{C} 13$ & $116.5(2)$ \\
\hline $\mathrm{N} 1-\mathrm{C} 1-\mathrm{H} 1 \mathrm{~A}$ & 108.4 & $\mathrm{C} 8-\mathrm{C} 9-\mathrm{H} 9$ & 118.8 \\
\hline $\mathrm{N} 1-\mathrm{C} 1-\mathrm{C} 2$ & $106.87(18)$ & $\mathrm{C} 8-\mathrm{C} 9-\mathrm{C} 10$ & $122.4(2)$ \\
\hline $\mathrm{N} 1-\mathrm{C} 1-\mathrm{C} 6$ & 113.39 (19) & $\mathrm{C} 10-\mathrm{C} 9-\mathrm{H} 9$ & 118.8 \\
\hline $\mathrm{C} 2-\mathrm{C} 1-\mathrm{H} 1 \mathrm{~A}$ & 108.4 & $\mathrm{C} 9-\mathrm{C} 10-\mathrm{C} 14$ & $120.6(3)$ \\
\hline $\mathrm{C} 6-\mathrm{C} 1-\mathrm{H} 1 \mathrm{~A}$ & 108.4 & $\mathrm{C} 11-\mathrm{C} 10-\mathrm{C} 9$ & $117.9(2)$ \\
\hline $\mathrm{C} 6-\mathrm{C} 1-\mathrm{C} 2$ & $111.36(17)$ & $\mathrm{C} 11-\mathrm{C} 10-\mathrm{C} 14$ & $121.5(2)$ \\
\hline $\mathrm{N} 2-\mathrm{C} 2-\mathrm{C} 1$ & $113.59(18)$ & $\mathrm{C} 10-\mathrm{C} 11-\mathrm{H} 11$ & 118.5 \\
\hline $\mathrm{N} 2-\mathrm{C} 2-\mathrm{H} 2$ & 107.1 & $\mathrm{C} 10-\mathrm{C} 11-\mathrm{C} 12$ & $123.0(2)$ \\
\hline $\mathrm{N} 2-\mathrm{C} 2-\mathrm{C} 3$ & $109.98(18)$ & $\mathrm{C} 12-\mathrm{C} 11-\mathrm{H} 11$ & 118.5 \\
\hline $\mathrm{C} 1-\mathrm{C} 2-\mathrm{H} 2$ & 107.1 & $\mathrm{C} 7-\mathrm{C} 12-\mathrm{C} 15$ & $125.9(2)$ \\
\hline $\mathrm{C} 3-\mathrm{C} 2-\mathrm{C} 1$ & $111.70(18)$ & $\mathrm{C} 11-\mathrm{C} 12-\mathrm{C} 7$ & $117.7(2)$ \\
\hline $\mathrm{C} 3-\mathrm{C} 2-\mathrm{H} 2$ & 107.1 & $\mathrm{C} 11-\mathrm{C} 12-\mathrm{C} 15$ & $116.5(2)$ \\
\hline $\mathrm{C} 2-\mathrm{C} 3-\mathrm{H} 3 \mathrm{~A}$ & 109.1 & $\mathrm{C} 8-\mathrm{C} 13-\mathrm{H} 13 \mathrm{~A}$ & 109.5 \\
\hline $\mathrm{C} 2-\mathrm{C} 3-\mathrm{H} 3 \mathrm{~B}$ & 109.1 & $\mathrm{C} 8-\mathrm{C} 13-\mathrm{H} 13 \mathrm{~B}$ & 109.5 \\
\hline $\mathrm{C} 2-\mathrm{C} 3-\mathrm{C} 4$ & $112.32(19)$ & $\mathrm{C} 8-\mathrm{C} 13-\mathrm{H} 13 \mathrm{C}$ & 109.5 \\
\hline $\mathrm{H} 3 \mathrm{~A}-\mathrm{C} 3-\mathrm{H} 3 \mathrm{~B}$ & 107.9 & $\mathrm{H} 13 \mathrm{~A}-\mathrm{C} 13-\mathrm{H} 13 \mathrm{~B}$ & 109.5 \\
\hline $\mathrm{C} 4-\mathrm{C} 3-\mathrm{H} 3 \mathrm{~A}$ & 109.1 & $\mathrm{H} 13 \mathrm{~A}-\mathrm{C} 13-\mathrm{H} 13 \mathrm{C}$ & 109.5 \\
\hline $\mathrm{C} 4-\mathrm{C} 3-\mathrm{H} 3 \mathrm{~B}$ & 109.1 & $\mathrm{H} 13 \mathrm{~B}-\mathrm{C} 13-\mathrm{H} 13 \mathrm{C}$ & 109.5 \\
\hline $\mathrm{C} 3-\mathrm{C} 4-\mathrm{H} 4 \mathrm{~A}$ & 109.4 & $\mathrm{C} 10-\mathrm{C} 14-\mathrm{H} 14 \mathrm{~A}$ & 109.5 \\
\hline $\mathrm{C} 3-\mathrm{C} 4-\mathrm{H} 4 \mathrm{~B}$ & 109.4 & $\mathrm{C} 10-\mathrm{C} 14-\mathrm{H} 14 \mathrm{~B}$ & 109.5 \\
\hline $\mathrm{C} 3-\mathrm{C} 4-\mathrm{C} 5$ & $111.02(19)$ & $\mathrm{C} 10-\mathrm{C} 14-\mathrm{H} 14 \mathrm{C}$ & 109.5 \\
\hline $\mathrm{H} 4 \mathrm{~A}-\mathrm{C} 4-\mathrm{H} 4 \mathrm{~B}$ & 108.0 & $\mathrm{H} 14 \mathrm{~A}-\mathrm{C} 14-\mathrm{H} 14 \mathrm{~B}$ & 109.5 \\
\hline $\mathrm{C} 5-\mathrm{C} 4-\mathrm{H} 4 \mathrm{~A}$ & 109.4 & $\mathrm{H} 14 \mathrm{~A}-\mathrm{C} 14-\mathrm{H} 14 \mathrm{C}$ & 109.5 \\
\hline $\mathrm{C} 5-\mathrm{C} 4-\mathrm{H} 4 \mathrm{~B}$ & 109.4 & $\mathrm{H} 14 \mathrm{~B}-\mathrm{C} 14-\mathrm{H} 14 \mathrm{C}$ & 109.5 \\
\hline
\end{tabular}




$\begin{array}{ll}\mathrm{C} 4-\mathrm{C} 5-\mathrm{H} 5 \mathrm{~A} & 109.5 \\ \mathrm{C} 4-\mathrm{C} 5-\mathrm{H} 5 \mathrm{~B} & 109.5 \\ \mathrm{H} 5 \mathrm{~A}-\mathrm{C} 5-\mathrm{H} 5 \mathrm{~B} & 108.1 \\ \mathrm{C} 6-\mathrm{C} 5-\mathrm{C} 4 & 110.51(19) \\ \mathrm{C} 6-\mathrm{C} 5-\mathrm{H} 5 \mathrm{~A} & 109.5 \\ \mathrm{C} 6-\mathrm{C} 5-\mathrm{H} 5 \mathrm{~B} & 109.5 \\ & \\ \mathrm{~S} 1-\mathrm{N} 1-\mathrm{C} 1-\mathrm{C} 2 & -156.04(17) \\ \mathrm{S} 1-\mathrm{N} 1-\mathrm{C} 1-\mathrm{C} 6 & 80.9(2) \\ \mathrm{S} 1-\mathrm{C} 7-\mathrm{C} 8-\mathrm{C} 9 & 177.22(17) \\ \mathrm{S} 1-\mathrm{C} 7-\mathrm{C} 8-\mathrm{C} 13 & -0.7(3) \\ \mathrm{S} 1-\mathrm{C} 7-\mathrm{C} 12-\mathrm{C} 11 & -175.90(18) \\ \mathrm{S} 1-\mathrm{C} 7-\mathrm{C} 12-\mathrm{C} 15 & 4.4(3) \\ \mathrm{O} 1-\mathrm{S} 1-\mathrm{N} 1-\mathrm{C} 1 & -46.1(2) \\ \mathrm{O} 1-\mathrm{S} 1-\mathrm{C} 7-\mathrm{C} 8 & -173.93(17) \\ \mathrm{O} 1-\mathrm{S} 1-\mathrm{C} 7-\mathrm{C} 12 & 4.7(2) \\ \mathrm{O} 2-\mathrm{S} 1-\mathrm{N} 1-\mathrm{C} 1 & -174.73(18) \\ \mathrm{O} 2-\mathrm{S} 1-\mathrm{C} 7-\mathrm{C} 8 & -44.7(2) \\ \mathrm{O} 2-\mathrm{S} 1-\mathrm{C} 7-\mathrm{C} 12 & 133.97(19) \\ \text { N1-S1-C7-C8 } & 68.36(19) \\ \text { N1-S1-C7-C12 } & -112.99(19) \\ \text { N1-C1-C2-N2 } & 57.9(2) \\ \text { N1-C1-C2-C3 } & -176.97(18) \\ \text { N1-C1-C6-C5 } & 175.8(2) \\ \text { N2-C2-C3-C4 } & 179.90(18) \\ \text { C1-C2-C3-C4 } & 52.8(3) \\ & \\ & \end{array}$

$\mathrm{C} 12-\mathrm{C} 15-\mathrm{H} 15 \mathrm{~A}$
$\mathrm{C} 12-\mathrm{C} 15-\mathrm{H} 15 \mathrm{~B}$
$\mathrm{C} 12-\mathrm{C} 15-\mathrm{H} 15 \mathrm{C}$
$\mathrm{H} 15 \mathrm{~A}-\mathrm{C} 15-\mathrm{H} 15 \mathrm{~B}$
$\mathrm{H} 15 \mathrm{~A}-\mathrm{C} 15-\mathrm{H} 15 \mathrm{C}$
$\mathrm{H} 15 \mathrm{~B}-\mathrm{C} 15-\mathrm{H} 15 \mathrm{C}$
$\mathrm{C} 2-\mathrm{C} 1-\mathrm{C} 6-\mathrm{C} 5$
$\mathrm{C} 2-\mathrm{C} 3-\mathrm{C} 4-\mathrm{C} 5$
$\mathrm{C} 3-\mathrm{C} 4-\mathrm{C} 5-\mathrm{C} 6$
$\mathrm{C} 4-\mathrm{C} 5-\mathrm{C} 6-\mathrm{C} 1$
$\mathrm{C} 6-\mathrm{C} 1-\mathrm{C} 2-\mathrm{N} 2$
$\mathrm{C} 6-\mathrm{C} 1-\mathrm{C} 2-\mathrm{C} 3$
$\mathrm{C} 7-\mathrm{S} 1-\mathrm{N} 1-\mathrm{C} 1$
$\mathrm{C} 7-\mathrm{C} 8-\mathrm{C} 9-\mathrm{C} 10$
$\mathrm{C} 8-\mathrm{C} 7-\mathrm{C} 12-\mathrm{C} 11$
$\mathrm{C} 8-\mathrm{C} 7-\mathrm{C} 12-\mathrm{C} 15$
$\mathrm{C} 8-\mathrm{C} 9-\mathrm{C} 10-\mathrm{C} 11$
$\mathrm{C} 8-\mathrm{C} 9-\mathrm{C} 10-\mathrm{C} 14$
$\mathrm{C} 9-\mathrm{C} 10-\mathrm{C} 11-\mathrm{C} 12$
$\mathrm{C} 10-\mathrm{C} 11-\mathrm{C} 12-\mathrm{C} 7$
$\mathrm{C} 10-\mathrm{C} 11-\mathrm{C} 12-\mathrm{C} 15$
$\mathrm{C} 12-\mathrm{C} 7-\mathrm{C} 8-\mathrm{C} 9$
$\mathrm{C} 12-\mathrm{C} 7-\mathrm{C} 8-\mathrm{C} 13$
$\mathrm{C} 13-\mathrm{C} 8-\mathrm{C} 9-\mathrm{C} 10$
$\mathrm{C} 14-\mathrm{C} 10-\mathrm{C} 11-\mathrm{C} 12$

109.5

109.5

109.5

109.5

109.5

109.5

$55.2(2)$

$-55.0(3)$

$56.8(3)$

$-57.3(3)$

$-177.74(19)$

$-52.6(2)$

$70.4(2)$

$-1.4(3)$

$2.7(3)$

$-177.0(2)$

2.8 (4)

$-176.1(2)$

$-1.4(4)$

$-1.3(4)$

$178.4(2)$

$-1.5(3)$

$-179.4(2)$

$176.7(2)$

$177.4(2)$

Hydrogen-bond geometry $\left(A,{ }^{\circ}\right)$

\begin{tabular}{lllll}
\hline$D-\mathrm{H} \cdots A$ & $D-\mathrm{H}$ & $\mathrm{H} \cdots A$ & $D \cdots A$ & $D-\mathrm{H} \cdots A$ \\
\hline $\mathrm{N} 2-\mathrm{H} 2 A \cdots \mathrm{N} 1$ & $0.89(3)$ & $2.43(3)$ & $2.877(3)$ & $111(2)$ \\
$\mathrm{N} 1-\mathrm{H} 1 \cdots \mathrm{N} 2^{\mathrm{i}}$ & $0.79(3)$ & $2.14(3)$ & $2.921(3)$ & $170(3)$ \\
\hline
\end{tabular}

Symmetry code: (i) $x-1 / 2,-y+3 / 2,-z+1$. 